\title{
DETECTION OF SKIN DIASEASE USING CURVLETS
}

\author{
Y.P.Gowramma ${ }^{1}$, Pavithra.. ${ }^{2}$, Manasa.S.B $^{3}$, Peetambari.B.P ${ }^{4}$, Vimala ${ }^{5}$ \\ ${ }^{1}$ Department of Computer Science \& Engineering, Kalpataru Institute of Technology, Tiptur-572202 \\ ${ }^{2}$ Department of Computer Science \& Engineering, Kalpataru Institute of Technology, Tiptur-572202 \\ ${ }^{3}$ Department of Computer Science \& Engineering, Kalpataru Institute of Technology, Tiptur-572202 \\ ${ }^{4}$ Department of Computer Science \& Engineering, Kalpataru Institute of Technology, Tiptur-572202 \\ ${ }^{5}$ Department of Computer Science \& Engineering, Kalpataru Institute of Technology, Tiptur-572202
}

\begin{abstract}
In this paper, we have proposed an algorithmic model for automatic classification of skin disease using Curvelet filter along with the $k$-nn classifier. The proposed algorithmic model is based on textural features such as curvelet filter responses. A skin disease is segmented using a Marker-Controlled Watershed Segmentation method. The dataset has different skin diseases with similar appearance (small inter class variations) across different classes and varying appearance (large intra class variations) within a class. Also, the images of diseases are of different pose with cluttered background.
\end{abstract}

Keywords: skin disease, curvelets, K-nn classifier.

\section{INTRODUCTION}

In the image processing and computer vision color, shape, and texture features are more important. But texture based analysis is very important in surface analysis. Skin is the surface of the body having some texture, diseased skin has variation in the texture of the skin. So we have proposed curvelet based texture analysis. A skin infection is an infection of the skin. Infection of the skin is distinguished from dermatitis which is inflammation of the skin, but a skin infection can result in skin inflammation. Skin inflammation due to skin infection is called infective dermatitis.

Human skin is a complex surface, with fine scale geometry that makes its appearance difficult to model. Melanin and hemoglobin pigments are contained in this structure. Slight changes of pigment construction in skin may cause a rich variation in skin color. By analyzing the skin texture, a lot of observations can be made regarding the nature and coarseness of the skin. Skin diseases, if not treated earlier might lead to severe complications in the body including spreading of the infection from one individual to the other. So it is necessary to be cautious regarding skin care. Developing a system for classification of skin disease is a difficult task because of considerable similarities among different classes and also due to a large intra-class variation. All these problems lead to a confusion across classes and make the task of skin disease identification more challenging. Applications of identification of images can be found useful in medical applications, disease analysis etc. Texture analysis is one of the fundamental aspects of human vision by which we discriminate between surfaces and objects. In the field of digital image processing, computer vision techniques can take advantage of the cues provided by surface texture to distinguish and recognize objects. Texture refers to visual patterns or spatial arrangement of pixels that regional intensity or color alone cannot sufficiently describe.

\section{LITERATURE SURVEY}

Many methodologies have been proposed to analyze and recognize textures in an automated fashion. In [3] A.C.Boviket. al. proposes a computational approach for analyzing visible textures by localizing spatial changes in the frequency, orientation, or phase of the textures using 2-D . Information extracted from the Curvelets responses are used to detect phase discontinuities within a texture. In [4] Haralick introduced a statistical and structural method to model texture based patterns based on the symmetric Grey Level Cooccurrence Matrix (GLCM). GLCM defines the probability of one grey tone occurring in the neighborhood of another grey tone at a specified distance and along a specified direction. Authors like Tamura [12] made an attempt at defining a set of visually relevant texture features which includes coarseness, contrast and directionality. Coarseness is the measure of granularity of an image, or average size of regions that have the same intensity, contrast is the measure of vividness of the texture pattern affected by the use of varying black and white intensities, directionality is the measure of directions of the grey values within the image. In [6] Lepisto proposed a method to retrieve non-homogenous, directional texture features based on texture Anal Kumar Mittra et al. / International Journal of Engineering Science and Technology [13] A. M. S. SMITH, M. J. WOOSTER, A. K. POWELL and D. USHER study aimed to investigate the potential of texturebased GLCM techniques for the identification of burn scars in low spatial resolution EO imagery since most current burn scar mapping techniques rely solely on pixel intensity 
information. The main focus of attention for texture-based classification in $\mathrm{EO}$ has been in determining the internal pixel structure of image regions since in general these methods fail to detect convoluted edges and small isolated features (Warner) 1990. Many methodologies have been proposed to analyze and recognize textures in an automated fashion. Tsumura et al [1] proposed a technique through which melanin and hemoglobin pigment content is extracted from a single skin color image by independent component analysis (ICA).

\section{PROPOSED METHOD}

The proposed method has training and classification phases. In training phase, from a given set of training images the texture features (Curlvlets) are extracted and used to train the system using the K-nearest neighbor classifier. In classification phase a given test infected diseased images is segmented using gradient magnitude method and then the above mentioned texture features are extracted for classification using Curvelets As mentioned above, Curvelets have the ability to perform multi-resolution decomposition due to its localization both in spatial and spatial frequency domain. Texture segmentation requires simultaneous measurements in both the spatial and the spatial-frequency domains. Filters with Smaller band width in the spatial-frequency domain are more desirable because they allow us to figure Make finer distinctions among different textures. These features are queried to K-nearest neighbor classifier to label an unknown disease.

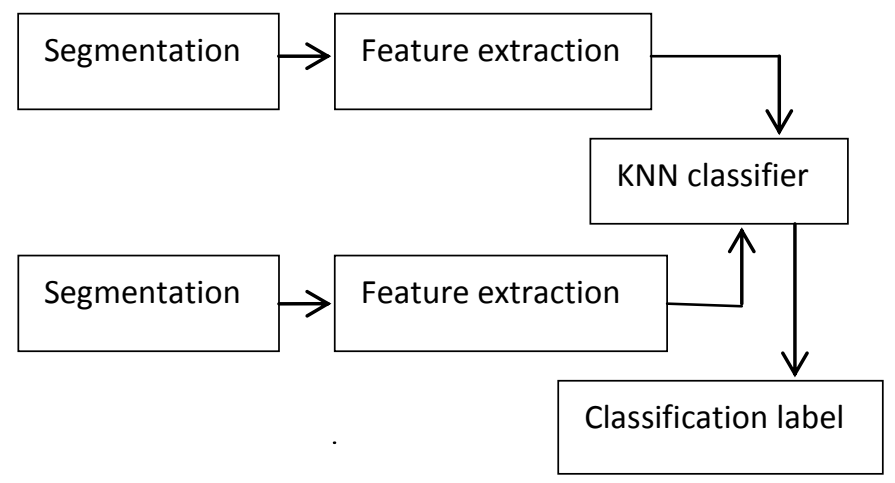

Fig 1: Proposed methodology

\subsection{Image Segmentation}

\section{Marker Controlled Water Shed Segmentation:}

Image segmentation is the process of partitioning an image into multiple segments, so as to change the representation of an image into something that is more meaningful and easier to analyze. The first step in skin disease classification is to segment the infected part of the image. Segmentation subdivides an image into its constituent parts or objects. In general, autonomous segmentation is one of the most difficult tasks in image processing in our project we segment the
Skin disease image using marker controlled water shed segmentation. This segmentation method fallows some basic procedures they are

- Compute a segmentation function. This is an image whose dark regions are the objects you are trying to segment.

- Compute foreground markers. These are connected blobs of pixels within each of the objects.

- Compute background markers. These are pixels that are not part of any object.

- Modify the segmentation function so that it only has minima at the foreground and background marker locations.

- Compute the watershed transform of the modified segmentation function.

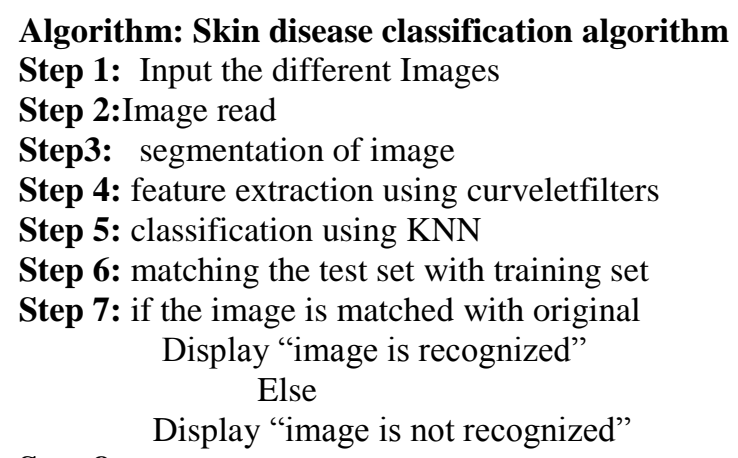

Step 8: stop.

\subsection{Feature Extraction}

\section{Curvelets}

Curvelets are a non-adaptive technique for multi-scale object representation. Being an extenction of the wavelet concept, They are becoming popular in similar fields, namely in image processing and scientific computing. Curvelets are an appropriate basic for representing images which are smooth apart from singularities along smooth curves, where the curves have bounded curvature, i.e. where objects in the image have a minimum length scale.

This property holds for cartoons, geometrical diagrams, and text. As one zooms in such images, the edges they contain appear increasingly straight. Curvelets take advantage of this property, by defining the higher resolution curvelets to be more elongated than the lower resolution curvelets. The curvelet transform is a multiscale directional transform that allows an almost optimal nonadaptive sparse representation of objects with edges. It has generated

Increasing interest in the community of applied mathematics and signal processing over the Years.

Curvelets are designed to handle curves using only a small number of coefficients.

Hence the CvT handles curve discontinuities well.

The four stages of the Curvelet Transform were: 
- Sub-band decomposition

- Smooth partitioning:

- Renormalization:

- Ridgelet analysis:

The Inverse of the Curvelet Transform:

- Ridgelet Synthesis

- Renormalization

- Smooth Integration

- Sub-band Recomposition.

Curvelets also exhibit an interesting architecture that sets them apart from classical multiscale representations. Curveletspartition the frequency plane into dyadic coronae and (unlike wavelets) subpartitions those into angular wedges which again display the parabolic aspect ratio. Hence, the curvelet transform refines the scale-space viewpoint by adding an extra element, orientation, and operates by measuring information about an object at specified scales and locations but only along specified orientations.

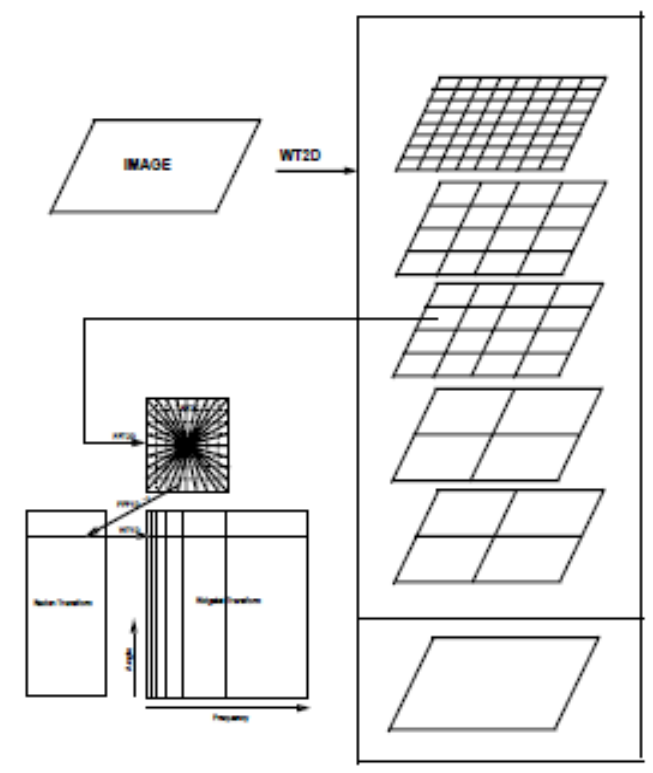

Fig2:Curvelet transform the figure illustrates the decomposition of theOriginal image into sub band followed by the spatial partitioning of each subband. The ridgelet transform is then applied to each block.

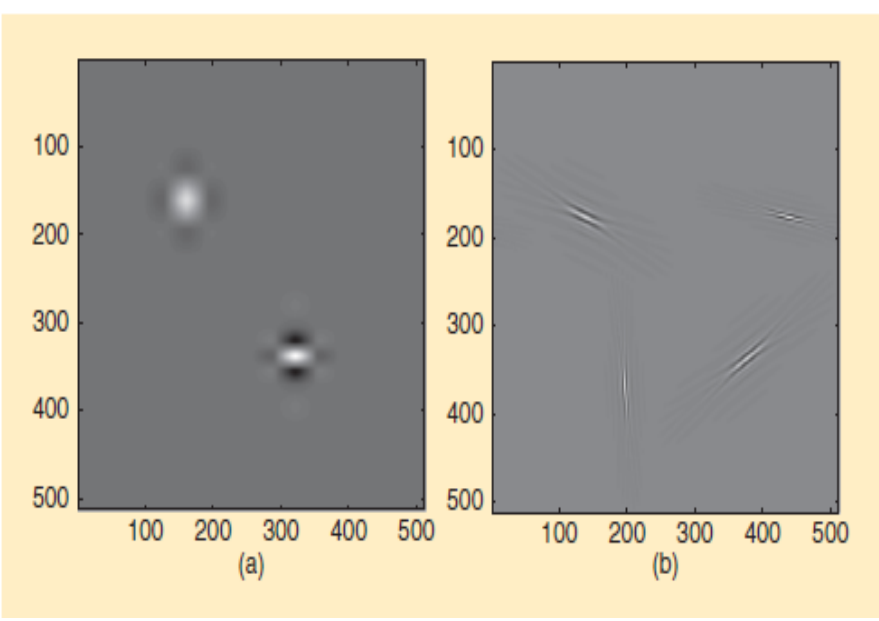

Fig 3:The elements of (a) wavelets and (b) curvelets on various scales, directions, andTranslations in the spatial domain. Note that the tensor-product 2-D wavelets are not strictly isotropic but prefer axes directions.

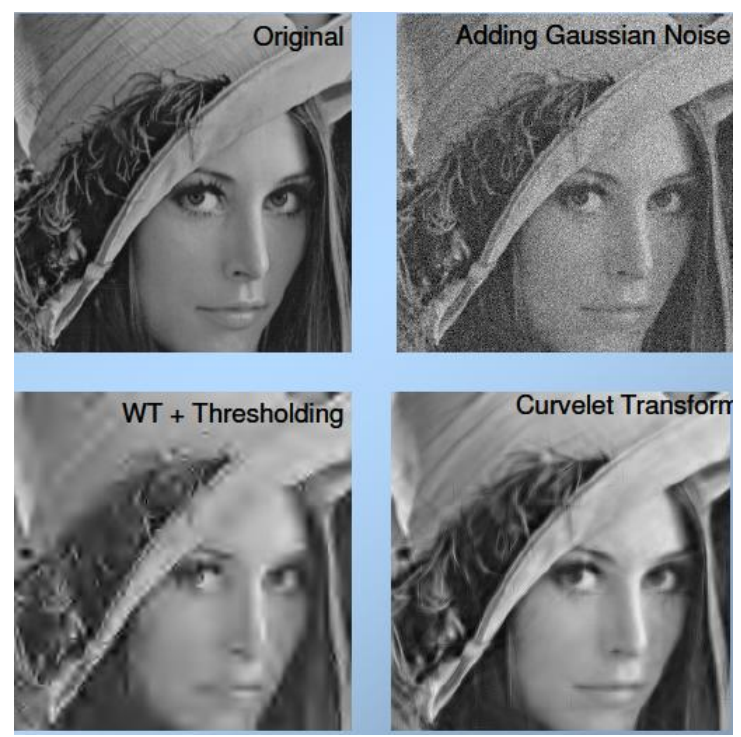

Fig 4:Enhancement using Curvelet transform.

\section{RESULTS AND DISCUSSION}

Figure 5 (figure 5.1 to figure 5.5) presents the different type of diseases infected to the skin in various parts. We have used the software MATLAB R2008a and the hardware 2GB RAM Intel core i3 to obtain the desired results. The curveletsand the inverse curvelets are applied on the diseased part of the skin and observed the resulting output image which gives good clarified region of the disease.

Figure 5:Diseased skin samples 


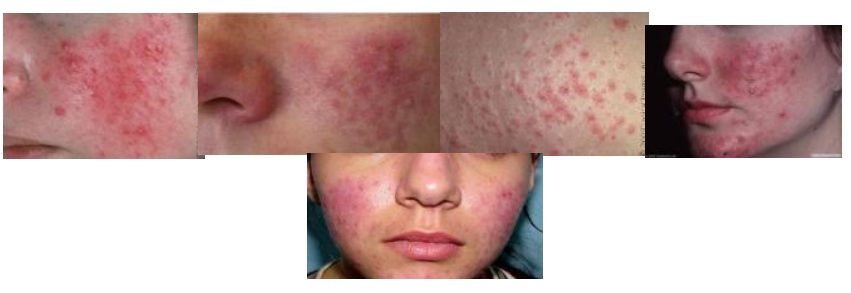

Fig 5.1:Rosasea

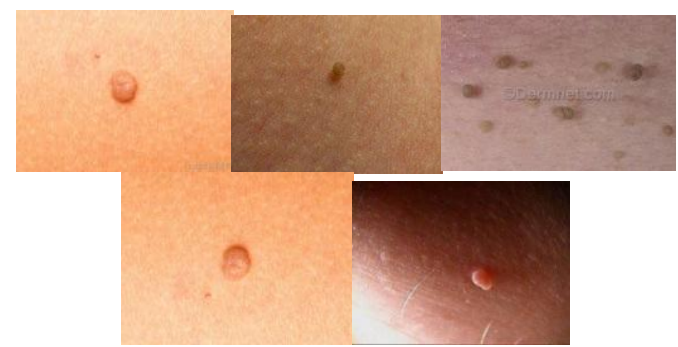

Fig5.2:Skintag

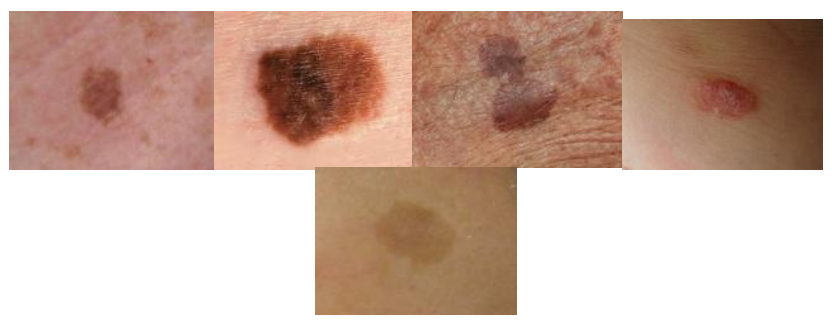

Fig 5.3:Liver spot

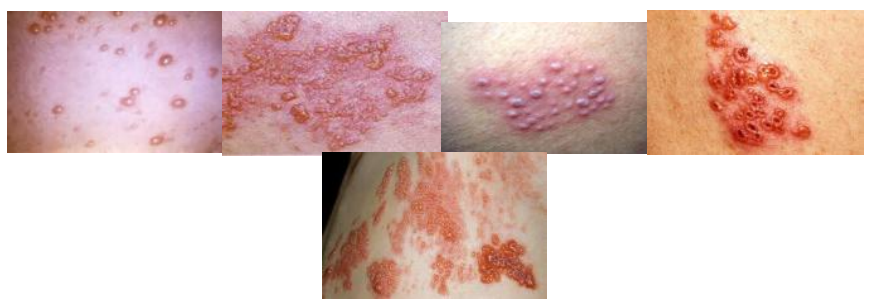

Fig 5.4: Shingales

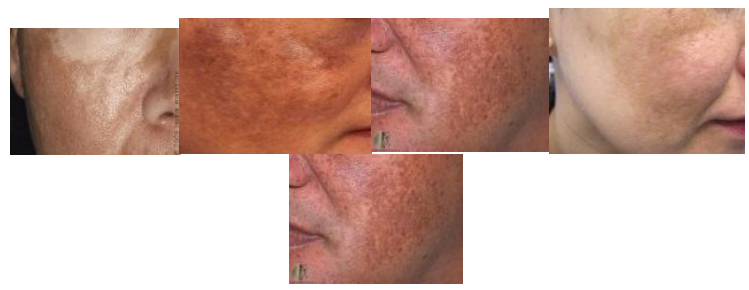

Fig 5.5:Melsama

\section{FUTURE ENHANCEMENT}

This application as of now can be used only with given set of images in a system. This work can be further done in the field of texture image segmentation. Moreover, for future work we can use various AI techniques like Radon neural network, Fuzzy, Adaptive, GA in order to attain the best output without performing calculations for each and every combination. This work can also be done using the technique of Gray Level Cooccurrence Matrix and using decision tree classifier. This current work only includes classification of normal skin diseases it can also be developed for classification of skin cancer like diseases.

\section{CONCLUSIONS}

The main focus of this paper is on analyzing the texture of skin thereby using it to diagnose the skin diseases. From the experimental results discussed above, we infer that the multiclass classification can serve as an effective tool in identifying skin diseases. The future work will be based on developing algorithms to identify various other skin diseases, to improve the overall efficiency and also to further reduce the computational time.

\section{REFERENCES}

[1].Al. Abadi, N. K.; Dahir, N. S.; Alkareem, Z. A. (2008): Skin texture recognition using neural network, in Proceedings of the International Arab Conference on Information Technology, Tunisia, December 16-18, pp.

[2].Blackledge, J. M.; Dubovitskiy, D. A. (2009): Texture classification using fractal geometry for the diagnosis of skin cancers, in Proceedings of EG UK Theory and Practice of Computer Graphics, UK, pp. 1-8.

[3].Bovik, A.C.; Clerk, M. and Geisler, W. S. (1990): Multichannel texture analysis using localized spatial filters, IEEE Transactions on Pattern Analysis \& Machine Intelligence, 12(1), pp. 55-73.

[4].Haralick, R.M. (1979): Statistical and structural approaches to Texture, Proceedings of IEEE, 67(5), pp. 784804.

[5].Kopec ,D.; Kabir, M. H.; Reinharth, D.; Rothschild ,O. and Castiglione ,J. A. (2003): Human errors in medical practice: systematic classification and reduction with automated information systems, Journal of Medical Systems, U K, 27(4), pp. $297-3$

[6].Rubegni, P. et al. (2002): Automated Diagnosis on Pigmented Skin Lesions, International Journal on Cancer, 101, pp. 576-580.

[7].Smach, F. et. al. (2006): Design of a neural network classifier for faceDetection, Journal of Computer Science, 2(3), pp. 257-260.

[8].Shyu, C. R.; Kak, A.; Kosaka, A. (1999): ASSERT a physician in the loop CBRS for HRCT image, databases, Comp. Vision and Image Understanding, 75(1), pp. 111-132.

[9].Tahmoush, D.; Samet, H. (2007): A Web collaboration system for content based retrieval of medical images, Proceedings of SPIE Medical Imaging - PACS and Medical Informatics, 6516, San Diego, USA.

[10].Tamura, H.; Mori, S.; Yamawaki, T. (1978): Textural Features Corresponding to Visual Perceptions, IEEE 
Transactions on Systems, Man and Cybernetics 8(6), pp. 460473.

[11]. Xia, S.; Mo, W.; Zhang , Z. (2005): A content based retrieval system for endoscopic images, Journal of Information Technology,11(2), pp. 27-32Anal Kumar Mittra et al. / International Journal of Engineering Science and Technology (IJEST)

[12].A. Bovik. Analysis of multichannel narrow-band filters for image texture segmentation.IEEE Transactions on Signal Processing, 39(9):2025-2043, 1991.

[13].F.J. Anscombe. The transformation of Poisson, binomial and negative-binomial data.Biometrika, 15:246\{254, 1948.

[14].A. Averbuch, R.R. Coifman, D.L. Donoho, M. Israeli, and J. Walden. Polar fft, rectopolarfft, and applications.Technical report, Stanford University, 2000.

[15].E. J. Candues. Harmonic analysis ofneuralnetworks.Applied and Computational Harmonic Analysis, 6:197\{218, 1999.

[16]. E. J. Candues. Monoscaleridgelets for the representation of images with edges.Technical report, Department of Statistics, Stanford University, 1999.Submitted for publication.

[17].E. J. Candues. On the representation of mutilated Sobolev functions.Technical report, Department of Statistics, Stanford University, 1999.Submitted for publication.

[18]. E. J. Candues and D. L. Donoho. Curvelets.Manuscript.http://wwwstat.stanford.edu/ donoho/R eports/1999/curvelets.pdf, 1999.

[19].E. J. Candues and D. L. Donoho. Curvelets $\{$ a surprisingly $\mathrm{e}^{\circledR e c t i v e n o n a d a p t i v e ~ r e p r e s e n t a t i o n ~ f o r ~ o b j e c t s ~}$ with edges. In A. Cohen, C. Rabut, and L.L. Schumacher, editors, Curve and Surface Fitting: Saint-Malo 1999, Nashville, TN, 1999. VanderbiltUniversity Press.

[20].E. J. Cand $\mu$ es and D. L. Donoho. Ridgelets: Key to Higher-dimensional Intermittency? Phil. Trans. R. Soc. Lond. A., 1999.

[21].E. J. Candues and D.L. Donoho. Edge-preserving denoising in linear inverse problems: Optimality of curvelet frames. Technical report, Department of Statistics, Stanford University, 2000.

[22].R.R. Coifman and D.L. Donoho. Translation invariant denoising.In A. Antoniadis and G. Oppenheim, editors, Wavelets and Statistics, pages $125\{150$, New York, 1995.SpringerVerlag.

[23].M. Crouse, R. Nowak, and R. Baraniuk. Wavelet-based statistical signal processing using hidden Markov models IEEE Transactions on Signal Processing, 46:886-902, 1998

[24]. USC-SIPI Image Database. http://sipi.usc.edu/services/database/-Database.html.

[25]. S. R. Deans. The Radon transform and some of its applications. John Wiley \& Sons, 1983.

[26]. M. N. Do and M. Vetterli. Orthonormal ${ }^{-}$niteridgelet transform for image compression.In Proc. of IEEE International Conference on Image Processing (ICIP), September 2000.
[27]. D. L. Donoho. Fast ridgelettransformsin dimension 2. Technical report, Stanford University, Department of Statistics, Stanford CA 94305-4065, 1997.

[28]. D. L. Donoho. Digital ridgelet transform via rectopolar coordinate transform. Technical report, Stanford University, 1998.

[29]. R.M. Mersereau and A.V. Oppenheim. Digital reconstruction of multidimensional signals from their projections.Proc .IEEE, 62(10):1319\{1338, 1974.

[30].T. Olson and J. DeStefano. Wavelet localization of the Radon transform. IEEE Trans.on Signal Process., 42(8):2055\{2067, 1994.

[31]. B. Sahiner and A.E. Yagle. On the use of wavelets in inverting the radon transform.InNuclear Science Symposium and Medical Imaging Conference, 1992., Conference Record of the 1992, volume 2, pages $1129\{1131,1992$.

[32].B. Sahiner and A.E. Yagle. Iterative inversion of the radon transform using imageadaptive wavelet constraints. In Engineering in Medicine and Biology Society, 1996. Bridging Disciplines for Biomedicine, 18th Annual International Conference of the IEEE, volume 2, pages 722\{723, 1997.

[33]. B. Sahiner and A.E. Yagle. Iterative inversion of the radon transform using imageadaptive wavelet constraints. In Image Processing,1998.ICIP98.Proceedings, volume2, pages 709\{713, 1998.

[34].E. P. Simoncelli. Bayesian denoising of visual images in the wavelet domain.InP Muller and B. Vidakovic, editors, Bayesian Inference in Wavelet Based Models. 